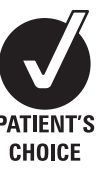

See Editorial Commentary, p 947

Department of Neurology, Hospital Universitario Ramón y Cajal, Madrid, Spain

Correspondence to Dr Jaime Masjuan, Department of Neurology, IRYCIS, Hospital Universitario Ramón y Cajal, Carretera de Colmenar, km 9.100, 28034 Madrid, Spain jmasjuan.hrc@salud.madrid.org

Received 1 February 2011 Revised 31 March 2011 Accepted 4 April 2011 Published Online First 7 May 2011

\title{
Neurology at the airport
}

\author{
Araceli Alonso-Cánovas, Alicia de Felipe-Mimbrera, Jaime González-Valcárcel, \\ Nuria García-Barragán, Iñigo Corral, Jaime Masjuan
}

\section{ABSTRACT}

Objectives Neurological problems are reported to be common in air travellers. The authors aimed to study neurological problems which might be associated with air traffic in a systematic way.

Methods The authors analysed a prospective registry of all the patients referred from Madrid-Barajas International Airport to the emergency department of their tertiary university hospital (Hospital Universitario Ramón y Cajal), for whom a neurological consultation was required, during a period of 21 months.

Results 77 patients with a history of air travel presented with neurological problems and were included in the analysis. Fifty-nine (76.6\%) were male, and the mean age was 45.9 (range 8-89, SD 17.5). Onset of symptoms was after landing in 44 subjects $(58.7 \%)$, during the flight in $31(41.3 \%)$, and unknown in two (5.1\%). Thirty-nine (50.9\%) had seizures, 18 (23.4\%) had a stroke, and $20(26 \%)$ other diagnosis. Sixty-one per cent of the patients with seizures had no previous history of epilepsy. Seizures on presentation were significantly associated with the use of drugs $(p=0.0008)$, and most of the cases with known epilepsy admitted nonadherence to treatment. Three 'body packers' were admitted with seizures secondary to intra-abdominal cocaine pack rupture. Of eight ischaemic strokes, five had high-grade carotid stenosis, and one case had economy-class stroke syndrome. Six patients with stroke were eligible and treated with intravenous thrombolysis. Conclusion In our series of neurological problems among air travellers, drug-induced seizures and ischaemic strokes due to large-artery atherosclerosis were the commonest observed diagnoses.

\section{INTRODUCTION}

Several studies have underscored the importance of in-flight medical emergencies. ${ }^{1-3}$ Among these, neurological problems are very frequent (around $30 \%$ of telemedicine calls), and only second to cardiovascular diseases regarding flight diversions. ${ }^{4}$ Recognition of symptoms and signs of neurological disease is critical for the airplane crew and medical volunteers, especially regarding time-dependent pathologies as stroke. Furthermore, flight diversions are costly for airplane companies, and cerebrovascular symptoms commonly misrecognised. ${ }^{5}$ This has led to increasing effort in airplane personnel training and implementation of telemedicine devices. Dizziness, seizures, headache and cerebrovascular diseases are the most common categories reported in in-flight medical emergencies registries. Economy-class stroke syndrome and airplane headache are newly described neurological entities specifically linked to the experience of travelling. ${ }^{6-8}$ Most of the scientific literature regarding neurological problems linked to air traffic consists of case reports or case series of a particular disease. Furthermore, they are mainly focused in problems arising during the flight, ignoring those presenting after landing We aimed to study the variety and incidence of all the neurological problems which might be associated with air traffic in a comprehensive and systematic way.

\section{METHODS}

Our centre is a tertiary university hospital and reference hospital for the international airport of the town, Madrid-Barajas, 11th in the Airports Council International ranking with an annual traffic of almost 50 million passengers (48 250784 ) in 2009. We prospectively collected data from all consecutive patients referred from the airport by airport medical services for whom a consultation with neurology was required, over a period of 21 months (from 1 May 2008 to 31 January 2010). Patients with a history of air travel of any duration, who developed neurological symptoms during the flight or within a maximum of $6 \mathrm{~h}$ after landing, were included. We excluded from the analysis any subjects who had no history of air travel, or were in the airport for different reasons (employees of the airport, relatives or friends of the travellers) in order to focus strictly on flight-related problems. All patients were assessed by a neurologist. Ancillary tests such as toxicology drug screening, brain computerised tomography scanning or magnetic resonance imaging were performed upon the neurologist's request. Patients with ischaemic stroke were admitted to the Stroke Unit, and treatment with intravenous standard-dose alteplase $(0.9 \mathrm{mg} / \mathrm{kg})$ was administered by the neurologist within $4.5 \mathrm{~h}$ of stroke onset. Patent foramen ovale (PFO) was routinely screened in all the patients with ischaemic stroke, by means of transcranial Doppler and echocardiography, with an injection of agitated saline during normal breathing and after a Valsalva manoeuvre. Economy-class stroke syndrome was diagnosed when PFO was demonstrated in a patient with an ischaemic stroke and a history of long immobilisation. For each subject, the following data were collected: demographic characteristics, past medical history (vascular risk factors and previous seizures), regular or recent use of recreational drugs, and flight history (duration of the flight and onset of symptoms during the flight). They were further classified regarding diagnostic categories: seizures, stroke and other diagnosis. Admission to an intensive care unit or stroke unit was noted. The type of stroke and aetiology were specified. The severity on admission and discharge (as measured by the National Institute of Health Stroke Scale (NIHSS) and Modified Rankin Scale 
(MRS), assessed by a neurologist $)^{9}$ and the application of a specific therapy (intravenous thrombolysis, surgery) were also noted. Seizures were classified on idiopathic or cryptogenic epileptic seizures, provoked or acute symptomatic seizures (when structural lesions, use or withdrawal of recreational drugs, or metabolic disturbances caused seizures), remote symptomatic seizures and psychogenic non-epileptic seizures (adapted from International League Against Epilepsy classification $^{10}$ ). Triggers of seizures (omission of antiepileptic drugs, sleep deprivation and use of recreational drugs) were recorded, as well as status epilepticus on presentation.

\section{Statistical methods}

We aimed to analyse whether past seizures, vascular risk factors, history of a long flight and use of drugs were associated with seizures or stroke. We performed a univariate analysis using a $\chi^{2}$ test for qualitative variables with a level of significance of $\mathrm{p}<0.05$.

\section{RESULTS}

Six hundred and twenty-one patients with medical problems were referred from the airport to our emergency department (ED). One hundred and fourteen required neurological consultation (18.35\%). Of these, 37 subjects without any history of air travel were excluded from analysis. Seventy-seven patients with a history of air travel and neurological problems remained for analysis.

Demographic features, past medical history, flight history and destination are summarised in table 1 . As for syndromic diagnosis, 39 subjects $(50.6 \%)$ had seizures, and 18 subjects had a stroke $(23.4 \%)$. Two patients with a stroke presented with seizures and were classified in both groups accordingly (see below). Twenty patients (26\%) had a different diagnosis. As for destination, 41 patients $(57.1 \%)$ were discharged from ED,

Table 1 Demographic factors, past medical history and flight history

\begin{tabular}{lllll}
\hline $\begin{array}{l}\text { Demographic and } \\
\text { clinical features }\end{array}$ & $\begin{array}{l}\text { All, } \\
\mathbf{N =}\end{array}$ & $\begin{array}{l}\text { Seizures, } \\
\text { (\%) }\end{array}$ & $\begin{array}{l}\text { Stroke, } \\
\mathbf{3 9 0 . 6 )}\end{array}$ & $\begin{array}{l}\mathbf{1 8}(\mathbf{2 3 . 4}) \\
\mathbf{2 0}(\mathbf{2 6})\end{array}$ \\
\hline Gender & & & & \\
Male & $59(76.6)$ & $31(79.5)$ & $14(77.8)$ & $14(70)$ \\
Female & $18(23.4)$ & $8(20.5)$ & $4(22.2)$ & $6(30)$ \\
Age: mean $\pm S D$ (range) & $45.9 \pm 17.5$ & $38.8 \pm 14.3$ & $60.7 \pm 15.5$ & $46.4 \pm 17.1$ \\
& $(8-89)$ & $(8-89)$ & $(24-83)$ & $(22-79)$ \\
Past medical history & & & & \\
Vascular risk factors & $34(44.2)$ & $10(25.7)$ & $14(77.8)^{*}$ & $10(50)$ \\
Past seizures & $19(24.7)$ & $16(41) \dagger$ & $1(5.5)$ & $2(10)$ \\
Drugs & $24(31.2)$ & $19(48.7) \neq$ & $3(16.7)$ & $2(10)$ \\
Alcohol & $18(23.4)$ & $14(36)$ & $3(16.7)$ & $1(5)$ \\
Cocaine & $6(7.8)$ & $4(10.3)$ & 0 & $2(10)$ \\
Cannabis & $2(2.6)$ & $2(5.1)$ & 0 & 0 \\
Sedatives & $2(2.6)$ & $2(5.1)$ & 0 & 0 \\
Amphetamines & $1(1.3)$ & $1(2.6)$ & 0 & 0 \\
Flight duration & & & & \\
$\geq 3$ h & $42(61.8)$ & $22(56.4)$ & $7(38.9)$ & $13(65)$ \\
$<3$ h & $26(38.2)$ & $11(28.2)$ & $8(44.4)$ & $7(35)$ \\
$\quad$ Unknown & $9(11.7)$ & $6(15.4)$ & $3(16.7)$ & 0 \\
Onset of symptoms & & & & \\
During the flight & $31(41.3)$ & $15(38.5)$ & $5(27.8)$ & $11(55)$ \\
After landing & $44(58.7)$ & $23(59)$ & $13(72.2)$ & $8(40)$ \\
Unknown & $2(5.1)$ & $1(2.6)$ & 0 & $1(5)$ \\
\hline
\end{tabular}

*Vascular risk factors were significantly associated with stroke $(p=0.0017)$ †Past seizures were significantly associated with seizures $(p=0.0007)$.

fUse of drugs was significantly associated with seizures $(p=0.0008)$.
$13(16.9 \%)$ were admitted to the Stroke Unit, $11(14.3 \%)$ to the Intensive Care Unit and nine (11.7\%) to Neurology wards.

\section{Seizures}

Forty-one patients $(53.2 \%$, including the two patients with stroke and seizures at the onset) presented with seizures. Twenty-five patients (61\%) had no previous history of seizures. Long flight duration $(53.6 \%)$ and onset of symptoms during the flight $(36.6 \%)$ were common. Nineteen patients $(56.6 \%)$ had a history of regular or recent use of recreational drugs, especially alcohol (14 patients, 34.1\%). Twenty-one patients (51.2\%) had a single seizure, 13 had more than one seizure (31.7\%), and seven cases $(17.1 \%)$ presented with status epilepticus. Final diagnoses are summarised in table 2. Among provoked or acute symptomatic seizures, most of the patients (12 of 13) had a history of drug use or withdrawal. Noteworthy is the case of three "body packers' (people who swallow drug-filled packets in order to smuggle illicit drugs) who presented with seizures secondary to cocaine-pack rupture with massive cocaine liberation. As for triggers of seizures, among the 13 patients with known epilepsy, 12 had a possible trigger for the seizure: 10 patients had missed one or more doses of antiepileptic drugs in the previous days, four had sleep deprivation, and three had used recreational drugs.

\section{Stroke}

Eighteen patients $(23.4 \%)$ were admitted with a stroke. Fourteen reported vascular risk factors: hypertension in 10, tobacco use in four, cardiopathy with high embolic risk in four, hypercholesterolaemia in three and diabetes mellitus in one. Eight patients had an ischaemic stroke (10.4\% of the sample). Severity on admission as measured by NIHSS was moderate (median 10 points, range 0-24). Stroke subtypes are detailed in table 3. Five patients had symptomatic high-grade carotid stenosis. There was a single case of economy-class stroke syndrome in a patient with history of long flight and immobilisation, massive PFO with curtain pattern and paradoxical thromboembolism without concomitant pulmonary embolism. Screening of PFO was negative in the remaining patients with ischaemic stroke. Six patients $(75 \%)$ were eligible for intravenous thrombolysis; the remaining two were not treated because of admission beyond the therapeutic window. The global outcome was favourable with a median NIHSS on discharge of 2 (range 0-18) and $75 \%$ independent patients (MRS 0-2).

Eight patients had an intracranial haemorrhage $(10.4 \%$ of the sample). Localisation was intraparenchymal in three, subdural in

Table 2 Aetiological classification of seizures (adapted from International League Against Epilepsy 2006)

\begin{tabular}{|c|c|}
\hline Seizures & $n=41(39+2) *(50.9 \%)$ \\
\hline Idiopathic or cryptogenic epileptic seizures & $20(48.8 \%)$ \\
\hline Provoked or acute symptomatic seizures & $16(39 \%)$ \\
\hline Drugs (use or withdrawal) & 12 \\
\hline Alcohol & 9 \\
\hline Cocaine (body packers) & 3 \\
\hline Acute ischaemic stroke & 2 \\
\hline Arteriovenous malformation & 1 \\
\hline Massive pulmonary embolism & 1 \\
\hline Spontaneous remote symptomatic seizures & $3(7.3 \%)$ \\
\hline Chronic cerebrovascular disease & 2 \\
\hline Neurocysticercosis & 1 \\
\hline Psychogenic non-epileptic seizures & $4(9.7 \%) \dagger$ \\
\hline
\end{tabular}


Table 3 Stroke subtypes and aetiology

\begin{tabular}{ll}
\hline Stroke & $\mathbf{n = 1 8 ~ ( 2 3 . 4 \% )}$ \\
\hline Transient ischaemic attack & 2 \\
Ischaemic stroke & 8 \\
Atherothrombotic over 90\% stenosis & 5 \\
Atherothrombotic below 50\% stenosis & 1 \\
Economy-class stroke & 1 \\
Undetermined aetiology & 1 \\
Intracranial bleeding & 8 \\
Primary (hypertensive, amyloid) & 3 \\
Traumatic brain injury & 2 \\
Aneurismatic & 1 \\
Oral anticoagulants & 1 \\
Idiopathic & 1 \\
\hline
\end{tabular}

two, subarachnoid in one and combined in the remaining three. Aetiological factors are detailed in table 3 . Three cases required surgical drainage and another case endovascular treatment. Global prognosis was poor with two deaths and three dependent patients (MRS 3-5) on discharge.

\section{Other diagnosis}

In 20 cases (26\%), an alternative diagnosis to seizures or stroke was made (table 4).

\section{Statistical analysis}

There was no association among long flights and final diagnosis of stroke or seizures. Use of recreational drugs and past seizures were significantly associated with seizures, and vascular risk factors with stroke (table 1).

\section{DISCUSSION}

Herein, we present a prospective series showing the variety and incidence of neurological disease associated with air traffic. Our sample is large and based on a representative population: an international airport with almost 50 million passengers a year. Since our hospital is the reference centre for medical emergencies at the airport, it is unlikely for us to overlook major medical problems occurring during the flight or after landing, with the exception, perhaps, of patients with onset of symptoms after leaving the airport. This is noteworthy, as most of the studies on medical and neurological problems linked to air traffic are solely focused on in-flight emergencies, while in our series, $58.7 \%$ arose after landing.

Table 4 Clinical features of patients with other diagnoses

\begin{tabular}{ll}
\hline Other diagnoses & $\mathbf{n = 2 0 ~ ( 2 6 \% ) ~}$ \\
\hline Drug toxicity (cocaine, zolpidem, carbamazepine, metformine) & 4 \\
Non-organic symptoms (except non-organic seizures) & 4 \\
Syncope & 2 \\
Peripheral neuropathy (radial and facial palsy) & 2 \\
Delirium in cognitively impaired & 2 \\
Neoplasms of central nervous system (primary CNS & 2 \\
lymphoma in HIV+; meduloblastoma*) & 1 \\
Viral meningoencephalitis & 1 \\
Chronic stroke (on demand for rehabilitation)* & 1 \\
Migraine with prolonged aura & 1 \\
Transient global amnesia & \\
\hline *Patients from developing countries. &
\end{tabular}

As suggested elsewhere, ${ }^{4}$ we confirm that neurological problems are very common in this setting, accounting for $18.35 \%$ of the patients transferred from the airport to our ED. The most frequent profile is a middle-aged male patient with a history of a long-haul flight, especially in patients presenting with seizures.

Seizures were the most common complaint in our group, and the use of recreational drugs was significantly associated with seizures. Interestingly, for the vast majority, this episode was the first seizure of their lives. We may therefore suggest that the use of drugs in the setting of long international flights (with sleep disruption and possibility of deprivation) is a risk factor for first seizures to occur in vulnerable subjects. Drug screening is mandatory in any patient with seizures or altered mental status of unknown origin in the setting of air traffic and may be critical for potentially fatal diagnoses as cocaine packs rupture. As for epileptic patients, a study by Trevorrow found that a previous high seizure frequency, fear of having a seizure and history of seizures in previous flights were the main factors in predicting a seizure following a flight. ${ }^{11}$ The use of drugs and sleep pattern were not investigated. In our experience, among the 13 epileptic patients who were admitted for a seizure, almost all patients reported a possible trigger, and non-adherence to antiepileptic drugs was strikingly frequent. This stresses the importance of the education of epileptic patients, who should be instructed to remain adherent and avoid other triggers for seizures in the vulnerable period of air travelling, especially if their disease is poorly controlled.

We found common causes of stroke to be most frequent in airtraffic-associated ischaemic stroke, with a surprisingly high proportion of high-grade carotid stenosis, while only one patient suffered a typical economy class stroke. It has been suggested that the hypobaric and hypoxic environment inside the aircraft may cause ischaemia in patients with reduced vascular reserve or arterial dissection. ${ }^{5}$ Even if the number of cases in our series is too low, we suggest that neurologists give some words of caution to patients with known high-grade stenosis who plan to fly, because of the potentially increased risk of stroke linked to air travel. On the other hand, in our series, prompt referral to the ED allowed treatment with intravenous thrombolysis in a large proportion of these patients, favouring a good outcome in most cases. Cooperation among the airport medical services, our hospital emergency medicine physicians and neurologists is essential in this setting and may be further promoted by the implementation of a stroke code, currently in use in the region of Madrid.

Finally, the subgroup of patients with other diagnoses is rather heterogeneous, with the toxicity of recreational and therapeutic drugs and non-organic symptoms being the most common diagnosis. Two patients sought medical attention presumably unavailable in their countries of origin. This phenomenon is not uncommon in a country such as Spain with universal healthcare, including care for tourists and non-residents. We did not record any cases of other diseases specifically linked to air traffic, recently described in scientific literature, namely airplane-headache related to atmospheric pressure changes. In addition, while other studies found syncope and dizziness to be the most common neurological complaints, ${ }^{2} 4$ these symptoms were rarely reported in our series. It is most likely that these patients were treated by airport medical services and emergency medicine physicians without neurological consultation.

A limitation of our study is the lack of information regarding flight diversions, or if therapeutic measures were applied on-board when the onset of symptoms was during the flight. Neurologists and other physicians on-board may be able to 


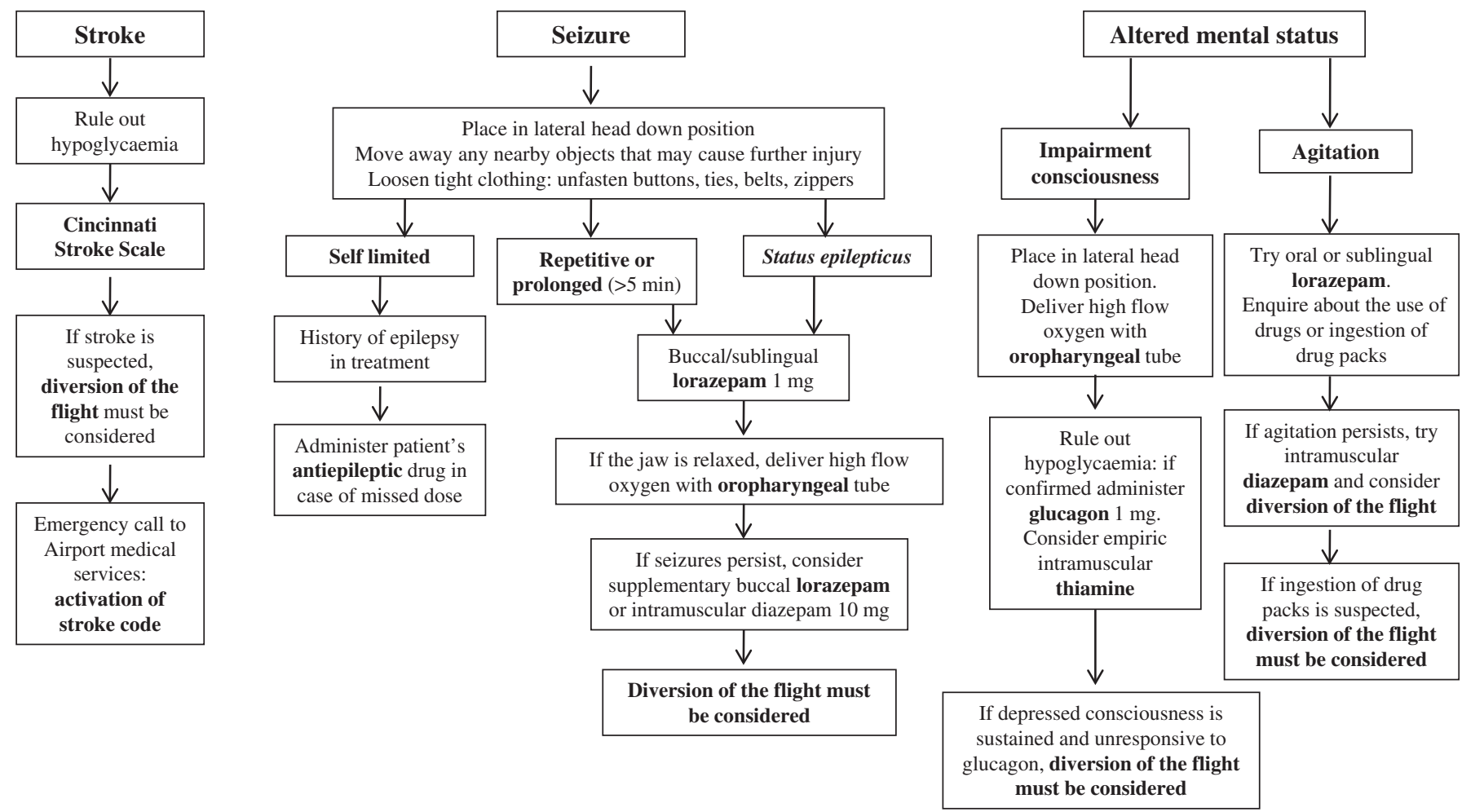

Figure 1 Proposed algorithm for the management of stroke, seizures and altered mental status on-board.

handle some serious neurological problems on-board (figure 1). In cases of repetitive or prolonged seizures and status epilepticus, a sublingual or buccal tablet of lorazepam, intranasal midazolam or rectal diazepam may be efficacious; these carry a smaller risk of central nervous system depression than intramuscular diazepam and are easier to administer. ${ }^{12}{ }^{13}$ In cases of impairment consciousness, a lateral head-down position may prevent aspiration and an oropharyngeal airway may be placed to deliver high-flow oxygen. Most medical kits on-board include oral or intramuscular antiemetics, analgesics and sedatives to treat nausea, pain and agitation. ${ }^{4}$ For agitation, we recommend using benzodiazepines instead of haloperidol, which may provoke serious adverse events in case of cocaine intoxication, such as seizures and extrapiramidal reactions (box 1).

As for stroke, it is critical for the aircrew (and, perhaps, for the physicians on-board) to recognise stroke symptoms and signs. Simplified stroke scales for non-physicians (such as the

\section{Box 1 Suggested medical kit for on-board neurological} problems

Oropharyngeal tube

- Capillary blood glucose test device and lancets

- Glucagon $1 \mathrm{mg} / \mathrm{ml}$

- Thiamine $100 \mathrm{mg} / \mathrm{ml}$

- Lorazepam (1 mg)

- Stesolid rectal tubes (diazepam, $10 \mathrm{mg} / 2 \mathrm{ml}$ )

- Intranasal midazolam $(0.2 \mathrm{mg} / \mathrm{kg}$, divided into each nare)

- Injectable diazepam (10 mg, $2 \mathrm{ml}$ )

- Metoclopramide tablets (10 mg) and injectable (10 mg, $2 \mathrm{ml})$

- Metamizole tablets $(500 \mathrm{mg})$ and injectable $(1 \mathrm{~g} / 2 \mathrm{ml})$

- Paracetamol tablets $(650 \mathrm{mg}, 1 \mathrm{~g})$
Cincinnati Stroke Scale) ${ }^{14}$ may be helpful for the training of aircraft personnel in this regard. In case of stroke, administration of antiplatelet or anticoagulant drugs is strongly discouraged. An emergency call to the airport medical services, with activation of a stroke code and prompt referral to the nearest Stroke Unit immediately after landing, may be time- and life-saving. Diversion of the flight or unscheduled landing must be considered on suspicion of stroke, altered mental status, and prolonged or multiple seizures.

This study suggests that air travel may induce a variety of neurological problems; seizures and stroke rank first and second, respectively, on the list. We observed an association among the use of recreational drugs and first seizures, and recognition of common triggers in seizures occurring in epileptic patients. We found a strikingly high proportion of high-grade carotid stenosis, whereas economy-class stroke syndrome was rare. Cooperation among airport medical services and in-hospital emergency services is crucial in time-dependent diseases as stroke.

Acknowledgements We wish to thank the staff of Barajas International Airport medical services (F Oliver and colleagues), for their crucial role in the attention of our patients and their essential cooperation. We thank Aeropuertos Españoles y Navegación Aérea, Servicios Aeroportuarios, Sección Relación con Compañías, Diomeda Ramírez Fernández, for providing information on the number of referrals from Barajas Airport to our emergency department. We also wish to thank AM Jiménez, an employee of Iberia Airlines (Iberia Líneas Aéreas de españa, SA), F Oliver, D Ramírez-Fernández and A Mimbrera for providing information regarding on-board medical kits and emergency procedures.

Funding AA-C received a grant from the Educational Federation of Neurological Societies (EFNS) educational fellowship in 2009.

Competing interests None.

Provenance and peer review Not commissioned; externally peer reviewed.

\section{REFERENCES}

1. Cummins RO, Schubach JA. Frequency and types of medical emergencies among commercial air travelers. JAMA 1989;261:1295-9. 
2. Weinlich M, Nieuwkamp N, Stueben U, et al. Telemedical assistance for in-flight emergencies on intercontinental commercial aircraft. J Telemed Telecare 2009;15:409-13

3. Chan SB, Hogan TM, Silva JC. Medical emergencies at a major international airport: in flight symptoms and ground-based follow-up. Aviat Space Environ Med 2002;73:1021-4.

4. Sirven JI, Claypool DW, Sahs KL, et al. Is there a neurologist on this flight? Neurology 2002;58:1739-44.

5. Leira EC, Cruz-Flores S, Wyrwich KW, et al. Improving pilot response to in-flight strokes: a randomized controlled trial. Cerebrovasc Dis 2005;19:317-22.

6. Kakkos SK, Geroulakos G. Economy class stroke syndrome: case report and review of the literature. Eur J Vasc Endovasc Surg 2004;27:239-43.

7. Belvís R, Masjuan J, García-Barragán N, et al. Stroke and pulmonary thromboembolism after a long flight. Eur J Neurol 2005;12:732-4.
8. Berilgen MS, Müngen B. Headache associated with airplane travel: report of six cases. Cephalalgia 2006;26:707-11.

9. Bonita R, Beaglehole R. Recovery of motor function after stroke. Stroke 1988:19:1497-500.

10. Engel J. ILAE classification core group. Epilepsia 2006;47:1558-68

11. Trevorrow T. Air travel and seizure frequency for individuals with epilepsy. Seizure 2006;15:320-7.

12. Appleton R, Macleod S, Martland T. Drug management for acute tonic-clonic convulsions including convulsive status epilepticus in children. Cochrane Database Syst Rev 2008;3:CD001905.

13. Holsti M, Sill BL, Firth SD, et al. Prehospital intranasal midazolam for the treatment of pediatric seizures. Pediatr Emerg Care 2007:23:148-53.

14. Kothari R, Hall K, Brott T, et al. Early stroke recognition: developing an out-of-hospital NIH Stroke Scale. Acad Emerg Med 1997:4:986-90.
Advancing Postgraduates. Enhancing Healthcare.

The Postgraduate Medical Journal is dedicated to advancing the understanding of postgraduate medical education and training.

- Acquire the necessary skills to deliver the highest possible standards of patient care

- Develop suitable training programmes for your trainees

- Maintain high standards after training ends

Published on behalf of the fellowship for Postgraduate Medicine

FOR MORE DETAILS OR TO SUBSCRIBE,

VISIT THE WEBSITE TODAY

postgradmedj.com 